\title{
Finite Alphabet Iterative Decoders, Part II: Towards Guaranteed Error Correction of LDPC Codes via Iterative Decoder Diversity
}

\author{
David Declercq, Senior Member, IEEE, Bane Vasic Fellow, IEEE, \\ Shiva Kumar Planjery, Student Member, IEEE, and Erbao Li
}

\begin{abstract}
Recently, we introduced a new class of finite alphabet iterative decoders (FAIDs) for low-density parity-check (LDPC) codes. These decoders are capable of surpassing belief propagation (BP) in the error floor region on the binary symmetric channel (BSC) with much lower complexity. In this paper, we introduce a novel scheme with the objective of guaranteeing the correction of a given and potentially large number of errors on column-weight-three LDPC codes. The proposed scheme uses a plurality of FAIDs which collectively correct more error patterns than a single FAID on a given code. The collection of FAIDs utilized by the scheme is judiciously chosen to ensure that individual decoders have different decoding dynamics and correct different error patterns. Consequently, they can collectively correct a diverse set of error patterns, which is referred to as decoder diversity. We provide a systematic method to generate the set of FAIDs for decoder diversity on a given code based on the knowledge of the most harmful trapping sets present in the code. Using the well-known column-weight-three $(155,64)$ Tanner code with $d_{\min }=20$ as an example, we describe the method in detail and show, by means of exhaustive simulation, that the guaranteed error correction capability on short length LDPC codes can be significantly increased with decoder diversity.
\end{abstract}

\section{INTRODUCTION}

It is now well established that iterative decoding based on belief propagation (BP) approaches the performance of maximum likelihood decoding (MLD) of the low density parity check (LDPC) codes asymptotically in the block length. However, for finite-length LDPC codes, the sub-optimality of iterative decoding manifests itself as the inability of the decoder to correct some low-noise configurations due to the presence of specific subgraphs in the Tanner graphs of the code, generically termed as trapping sets [1], [2]. The presence of trapping sets in a code gives rise to the error floor phenomenon which is an abrupt degradation in the error rate performance of the code in the high signal-tonoise ratio regime. This performance degradation has also been characterized by the notion of pseudo-codewords [3], which represent attractor points of iterative message-passing

This work was funded by the NSF under grant CCF-0963726 and the Institut Universitaire de France grant. Part of the material in this paper was presented at the 2012 Information Theory and Applications Workshop.

D. Declercq and E. Li are with ENSEA/University of CergyPontoise/CNRS UMR 8051, 95014 Cergy-Pontoise, France (email: \{declercq,erbao.li\}@ensea.fr). B. Vasic is with the Department of Electrical and Computer Engineering, University of Arizona, Tucson, AZ, 85719 USA (email: vasic@ece.arizona.edu). S. K. Planjery is with both the above institutions (email: shivap@ece.arizona.edu). decoders, analogous to codewords which are the solutions of the MLD. However, a precise structural relationship between trapping sets and pseudo-codewords of a given Tanner graph and a decoding algorithm is not yet fully established. It has also been pointed out by several works such as [4] that the minimum weight of pseudo-codewords is typically smaller that the minimum distance for most LDPC codes. Thus, the presence of trapping sets in the Tanner graph of the code in principle prevents the iterative decoders from approaching the performance of MLD for finite-length LDPC codes.

An LDPC code $\mathcal{C}$ is said to have a $t$-guaranteed error correction capability under a particular decoding algorithm over the binary symmetric channel (BSC) if it can correct all error patterns of weight $t$ or less. The guaranteed error correction capability of an LDPC code for the BSC plays a crucial role in its error floor performance as it determines the slope of the error floor [5]. Moreover, the problem of guaranteed error correction is critical for applications such as magnetic, optical and solid-state storage, flash memories, optical communication over fiber or free-space, as well as an important open problem in coding theory. Guaranteed error correction is typically achieved by using Bose-ChaudhuriHocquenghem $(\mathrm{BCH})$ or Reed-Solomon (RS) codes and harddecision decoders such as the Berlekamp-Massey decoder [6], but very little is known about the guaranteed error correction capability of LDPC codes under iterative decoding. The main reason for this comes from the fact that even though the error floor performance of an LDPC code can be relatively well characterized through the identification of its trapping sets, it is still an arduous task to determine whether a particular iterative decoder succeeds in correcting all $t$-error patterns. The guaranteed error correction capability of a particular LDPC code can vary depending on the particular iterative decoder that is being used [7].

In the first part of our two-part paper series, we introduced a new class of finite precision iterative decoders, referred to as finite alphabet iterative decoders (FAIDs) [8], [9], which are much lower in complexity than the BP algorithm but can provide a superior error-rate performance in the error floor region. FAIDs requiring only a small number of precision bits (as small as three) were shown to surpass BP in the error floor region on several codes of practical interest due to their ability to correct more low-weight error patterns than the BP algorithm [8], [10], [11]. For instance, on the column-weightthree $(155,64)$ Tanner code [12], it was shown that there are 
3-bit precision FAIDs that guarantee a correction of up to 5 errors, whereas the BP (implemented in floating-point with a maximum of 100 iterations) fails to correct several 5-error patterns [13].

Despite the superior error floor performance achieved by the FAIDs, their performance especially in terms of guaranteed error correction capability is still far from the performance of MLD. For example on the Tanner code, with its minimum distance $d_{\min }=20$, a guaranteed error correction of 5 errors achieved by FAIDs is still far from the capability of MLD which is 9 errors, therefore leaving large room for improvement.

In this paper, we aim at reducing this gap by introducing a general approach with the objective of improving the guaranteed error correction capability of LDPC codes. The approach relies on using a set of carefully chosen FAIDs which are tuned to have different dynamical behaviors in terms of their error correction on a given code. The idea is that if an error pattern cannot be corrected by one particular decoder, there is another decoder in the set that can correct this pattern. The set of selected FAIDs can then be used sequentially (that is, when a decoder fails to correct an error pattern, another decoder is used), to collectively correct a diverse set of error patterns including some which were not correctable by a single FAID. This capability of a set of FAIDs to collectively correct a diverse set of error patterns is referred to as decoder diversity. The framework of FAIDs and their simplicity makes them good candidates for decoder diversity as a plurality of FAIDs can easily be defined by specifying their variable node update maps.

The main objective of our approach can be summarized as follows: given a particular LDPC code, we would like to identify a set of FAIDs that when used sequentially can correct a fixed number of errors, say $t$. A brute force approach would rely on checking all possible error patterns up to weight $t$ for every FAID considered, and then choosing the set of FAIDs that correct all the patterns. However, this brute force approach would be prohibitively complex. Instead, we restrict our attention to only error patterns associated with the harmful topologies present in the code that could be trapping sets. Our approach then involves searching for such topologies in the code, considering all error patterns up to weight $t$ whose support lies in these topologies, and then finding a combination of FAIDs that can correct all these particular error patterns. In order to further reduce the computational complexity of our methodology, we make use of the group automorphisms of the Tanner graph of structured LDPC codes to reduce the number of considered topologies and associated error patterns. The automorphism group for a graph is the group of permutations that map nodes to nodes, edges to edges, and preserve edgenodes connections. As an example, for a quasi-cyclic LDPC code [14] with $(L \times L)$ circulant matrices, each trapping set has $L$ copies which are located elsewhere in the graph, but are all isomorphic in the sense that they have the exact same topological neighborhood and computation tree. As a result, the error patterns located on only one of the $L$ copies are sufficient to be considered for the selection of FAIDs. Using the quasi-cyclic $(155,64)$ Tanner code [12] as an example, we shall present our low complexity design methodology in detail and show, by means of exhaustive simulation, that the guaranteed error correction capability of this code can be increased from $t=5$ which is achievable by using a single FAID to $t=7$ by using FAID decoder diversity.

The rest of the paper is organized as follows. Section II provides the necessary preliminaries. Section III introduces the concept of decoder diversity and describes our general approach. In Section IV, we use the $(155,64)$ Tanner code as a case study and discuss in detail how our approach can be used to increase the guaranteed error correction capability of the code. Finally, conclusions are presented in Section V.

\section{PRELIMINARIES}

The Tanner graph $G$ of an $(N, K)$ binary LDPC code $\mathcal{C}$ is a bipartite graph with two sets of nodes: the set of variable nodes $V=\left\{v_{1}, \cdots, v_{N}\right\}$ and the set of check nodes $C=\left\{c_{1}, \cdots, c_{M}\right\}$. The set of neighbors of a node $v_{i}$ is denoted as $\mathcal{N}\left(v_{i}\right)$, and the set of neighbors of node $c_{j}$ is denoted by $\mathcal{N}\left(c_{j}\right)$. The degree of a node is the number of its neighbors. We shall consider only LDPC codes with regular column-weight $d_{v}$, where all variable nodes have the same degree $d_{v}$.

Let $\mathbf{x}=\left(x_{1}, x_{2}, \ldots, x_{N}\right)$ denote a codeword of $\mathcal{C}$ that is transmitted over the BSC, where $x_{i}$ denotes the value of the bit associated with variable node $v_{i}$, and let the channel output vector be denoted as $\mathbf{r}=\left\{r_{1}, r_{2}, \ldots, r_{N}\right\}$. Let $\mathbf{e}=\left(e_{1}, e_{2}, \ldots, e_{N}\right)$ be the error pattern introduced by the BSC such that $\mathbf{r}=\mathbf{x} \oplus \mathbf{e}$, and $\oplus$ is the modulo-two sum operator. The support of an error pattern e, denoted by $\operatorname{supp}(\mathbf{e})$, is defined as the set of all positions $i$ such that $e_{i} \neq 0$. The weight of the error pattern e, denoted by $w(\mathbf{e})$ is the cardinality of $\operatorname{supp}(\mathbf{e})$. Let $\mathbf{y}=\left(y_{1}, y_{2}, \ldots, y_{N}\right)$ denote the input vector to the decoder where each $y_{i}$, also referred to as a channel value, is calculated at a node $v_{i}$ based on the received value $r_{i}$.

\section{A. Finite Alphabet Iterative Decoders}

An $N_{s}$-level FAID [8] denoted by $\mathrm{D}$, is a 4-tuple given by $\mathrm{D}=\left(\mathcal{M}, \mathcal{Y}, \Phi_{v}, \Phi_{c}\right)$. The messages are levels confined to a finite alphabet $\mathcal{M}=\left\{-L_{s}, \ldots,-L_{2},-L_{1}, 0, L_{1}, L_{2}, \ldots, L_{s}\right\}$ consisting of $N_{s}=2 s+1$ levels, where $L_{i} \in \mathbb{R}^{+}$and $L_{i}>L_{j}$ for any $i>j$. The sign of a message $x \in \mathcal{M}$ can be interpreted as the estimate of the bit (positive for zero and negative for one) associated with the variable node to or from which $x$ is being passed, and the magnitude $|x|$ as a measure of how reliable this value is. The message 0 in the alphabet can be interpreted as an erasure message.

The set $\mathcal{Y}$ denotes the set of possible channel values. For the case of BSC, $\mathcal{Y}=\{ \pm \mathrm{C}\}$, where $\mathrm{C} \in \mathbb{R}^{+}$. By convention, we use the mapping $0 \rightarrow \mathrm{C}$ and $1 \rightarrow-\mathrm{C}$. Let $m_{1}, m_{2}, \ldots, m_{l-1}$ denote the $l-1$ extrinsic incoming messages of a node (check or variable) of degree $l$ which are used in the calculation of the outgoing message. 
The function $\Phi_{c}: \mathcal{M}^{d_{c}-1} \rightarrow \mathcal{M}$ is used for update at a check node with degree $d_{c}$ and is defined as

$$
\Phi_{c}\left(m_{1}, \ldots, m_{d_{c}-1}\right)=\left(\prod_{j=1}^{d_{c}-1} \operatorname{sgn}\left(m_{j}\right)\right) \min _{j \in\left\{1, \ldots, d_{c}-1\right\}}\left(\left|m_{j}\right|\right)
$$

The function $\Phi_{v}: \mathcal{Y} \times \mathcal{M}^{d_{v}-1} \rightarrow \mathcal{M}$ is a map used for update at a variable node with degree $d_{v}$.

It can described as a closed form function or simply as a $d_{v-1}$-dimensional array or look-up table (LUT). More details on the closed-form description are provided in the first part of our two-part series of papers [8]. In this paper, we shall only use the LUT form which is convenient for defining multiple update maps required for decoder diversity.

Note that the maps defining $\Phi_{v}$ must satisfy the symmetry property which is

$$
\Phi_{v}\left(y_{i}, m_{1}, \ldots, m_{d_{v}-1}\right)=-\Phi_{v}\left(-y_{i},-m_{1}, \ldots,-m_{d_{v}-1}\right)
$$

and the monotonicity property which is

$$
\Phi_{v}\left(-\mathrm{C}, m_{1}, \ldots, m_{d_{v}-1}\right) \geq \Phi_{v}\left(-\mathrm{C}, m_{1}^{\prime}, \ldots, m_{d_{v}-1}^{\prime}\right)
$$

when $m_{i} \geq m_{i}^{\prime} \forall i \in\left\{1, \ldots, d_{v}-1\right\}$.

Let us alternatively define $\mathcal{M}$ to be $\mathcal{M}=$ $\left\{M_{1}, M_{2}, \cdots, M_{N_{s}}\right\}$ where $M_{1}=-L_{s}, M_{2}=-L_{s-1}, \cdots$, $M_{s}=-L_{1}, M_{s+1}=0, M_{s+2}=L_{2}, \cdots, M_{N_{s}}=L_{s}$. For column-weight $d_{v}=3$ codes, the function $\Phi_{v}$ can be conveniently represented as a two-dimensional array $\left[l_{i, j}\right]_{1 \leq i \leq N_{s}, 1 \leq j \leq N_{s}}$, where $l_{i, j} \in \mathcal{M}$, such that $\Phi_{v}\left(M_{i}, M_{j},-\mathrm{C}\right)=l_{i, j}$ for any $M_{i}, M_{j} \in \mathcal{M}$. The values for $\Phi_{v}\left(M_{i}, M_{j},+\mathrm{C}\right)$ can be deduced from the symmetry of $\Phi_{v}$. The notations used for the LUT representation of $\Phi_{v}$ for a 7-level FAID are shown in Table I, and some examples are listed in Appendix B.

Table I

LUT REPRESENTATION OF $\Phi_{v}\left(-\mathrm{C}, m_{1}, m_{2}\right)$ FOR A 7-LEVEL FAID

\begin{tabular}{|c||c|c|c|c|c|c|c|}
\hline $\boldsymbol{m}_{\mathbf{1}} \boldsymbol{m}_{\mathbf{2}}$ & $-\boldsymbol{L}_{\mathbf{3}}$ & $-\boldsymbol{L}_{\mathbf{2}}$ & $-\boldsymbol{L}_{\mathbf{1}}$ & $\mathbf{0}$ & $\mathbf{+} \boldsymbol{L}_{\mathbf{1}}$ & $+\boldsymbol{L}_{\mathbf{2}}$ & $+\boldsymbol{L}_{\mathbf{3}}$ \\
\hline \hline $\boldsymbol{- \boldsymbol { L } _ { 3 }}$ & $l_{1,1}$ & $l_{1,2}$ & $l_{1,3}$ & $l_{1,4}$ & $l_{1,5}$ & $l_{1,6}$ & $l_{1,7}$ \\
\hline $\boldsymbol{- L}_{\mathbf{2}}$ & $l_{2,1}$ & $l_{2,2}$ & $l_{2,3}$ & $l_{2,4}$ & $l_{2,5}$ & $l_{2,6}$ & $l_{2,7}$ \\
\hline $\boldsymbol{- L}_{\mathbf{1}}$ & $l_{3,1}$ & $l_{3,2}$ & $l_{3,3}$ & $l_{3,4}$ & $l_{3,5}$ & $l_{3,6}$ & $l_{3,7}$ \\
\hline $\mathbf{0}$ & $l_{4,1}$ & $l_{4,2}$ & $l_{4,3}$ & $l_{4,4}$ & $l_{4,5}$ & $l_{4,6}$ & $l_{4,7}$ \\
\hline$+\boldsymbol{L}_{\mathbf{1}}$ & $l_{5,1}$ & $l_{5,2}$ & $l_{5,3}$ & $l_{5,4}$ & $l_{5,5}$ & $l_{5,6}$ & $l_{5,7}$ \\
\hline$+\boldsymbol{L}_{\mathbf{2}}$ & $l_{6,1}$ & $l_{6,2}$ & $l_{6,3}$ & $l_{6,4}$ & $l_{6,5}$ & $l_{6,6}$ & $l_{6,7}$ \\
\hline$+\boldsymbol{L}_{\mathbf{3}}$ & $l_{7,1}$ & $l_{7,2}$ & $l_{7,3}$ & $l_{7,4}$ & $l_{7,5}$ & $l_{7,6}$ & $l_{7,7}$ \\
\hline
\end{tabular}

\section{B. Trapping Sets}

A trapping set (TS) denoted by $\mathbf{T}$ for an iterative decoder is a non-empty set of variable nodes in $G$ that are not corrected at the end of a given number of iterations [1]. A standard notation commonly used to denote a trapping set is $(a, b)$, where $a=|\mathbf{T}|$, and $b$ is the number of odd-degree check nodes present in the subgraph induced by $\mathbf{T}$.

The Tanner graph representation of an $(a, b)$ TS denoted by $\mathcal{T}$ is a topological structure containing $a$ variable nodes and $b$ odd-degree check nodes. A code $\mathcal{C}$ is said to contain a TS of type $\mathcal{T}$ if there exists a set of variable nodes in $G$ whose induced subgraph is isomorphic to $\mathcal{T}$, seen as a topological structure. Let $N_{\mathcal{T}}$ denote the number of trapping sets of type $\mathcal{T}$ that are contained in the code $\mathcal{C}$. Note that we use $\mathbf{T}$ to refer to a particular subset of variable nodes in a given code that form a trapping set. Finally, let $\left\{\mathbf{T}_{i, \mathcal{T}} \mid i=1, \ldots, N_{\mathcal{T}}\right\}$ be the collection of trapping sets of type $\mathcal{T}$ present in code $\mathcal{C}$. In other words, $\left\{\mathbf{T}_{i, \mathcal{T}}\right\}_{i}$ is a collection of all distinct subsets of variable nodes whose induced subgraphs are isomorphic to trapping sets of type $\mathcal{T}$. A TS is said to be elementary if $\mathcal{T}$ contains only degree-one or/and degree-two check nodes. It is well known that the error floor phenomenon is associated with the presence of elementary trapping sets [1], [15]. Hence, throughout this paper, we shall only consider elementary trapping sets. Although the $(a, b)$ notation is typically used in the literature, this notation is not sufficient to uniquely denote a particular trapping set as there can be many trapping sets with different topological structures that share the same values of $a$ and $b$. This is important to consider since the topological structure of a particular $(a, b)$ TS determines how harmful the TS is for the error floor of a given decoder [2]. On the other hand, a notation which includes complete topological description of a subgraph would be extremely complicated and too precise for our purpose. Therefore, we introduce a simplified notation which only captures the cycle structure of the subgraph thus giving a cycle inventory of a trapping set.

Definition 1. Consider an $(a, b)$ trapping set whose corresponding subgraph consists of a variable nodes and $b$ odddegree check nodes. The trapping set is said to be of type $\left(a, b ; \prod_{k \geq 2}(2 k)^{g_{k}}\right)$ if the corresponding subgraph contains exactly $g_{k}$ distinct cycles of length $2 k$.

Our choice of notation appears to be sufficient for differentiating between the topological structures of multiple $(a, b)$ trapping sets, and also includes the definition of codewords of $\mathcal{C}$, as the $(a, 0)$ trapping sets correspond to codewords of weight $a$.

\section{DeCODER DiVERSITY}

\section{A. Decoder Diversity Principle}

We shall now formally introduce the concept of decoder diversity. Let us assume that we have at our disposal a set of $N_{s}$-level FAIDs denoted by

$$
\mathcal{D}=\left\{\left(\mathcal{M}, \mathcal{Y}, \Phi_{v}^{(i)}, \Phi_{c}\right) \mid i=1, \ldots, N_{\mathcal{D}}\right\}
$$

where each $\Phi_{v}^{(i)}$ is a uniquely defined map. We refer to this set $\mathcal{D}$ as a decoder diversity set with cardinality $N_{\mathcal{D}}$, and an element of this set is denoted by $\mathrm{D}_{i}$ where $\mathrm{D}_{i}=$ $\left(\mathcal{M}, \mathcal{Y}, \Phi_{v}^{(i)}, \Phi_{c}\right)$.

Given a code $\mathcal{C}$, we would like to determine whether the FAIDs in the set $\mathcal{D}$ could be used in combination in order to guarantee the correction of all error patterns up to a certain weight $t$. We first introduce the notation used to denote the set of error patterns correctable by a decoder. Let $\mathcal{E}$ denote an arbitrary set of error patterns that are considered for a code $\mathcal{C}$ 
whose Tanner graph is $G$, i.e., a set of vectors e with non-zero weight. Let $\mathcal{E}_{\mathrm{D}_{i}} \subseteq \mathcal{E}$ denote the subset of error patterns that are correctable by a FAID $\mathrm{D}_{i} \in \mathcal{D}$.

Definition 2. We say that the set of error patterns $\mathcal{E}$ is correctable by a decoder diversity set $\mathcal{D}$ if

$$
\mathcal{E}=\bigcup_{i=1}^{N_{\mathcal{D}}} \mathcal{E}_{\mathrm{D}_{i}}
$$

Note that at this point, we have not yet placed any limit on the maximum number of decoding iterations of each decoder $\mathrm{D}_{i}$, and this issue will be subsequently addressed in Section IV using the example of the $(155,64)$ Tanner code. Given a set of error patterns up to a certain weight $t$ on the code $\mathcal{C}$, one would like to determine the smallest decoder diversity set that can correct all such error patterns. This problem is known as the Set Covering Problem, and is NP-hard [16]. In this paper, we propose a greedy algorithm which can provide a decoder diversity set $\mathcal{D}$ of FAIDs that may not necessarily be the smallest set, but can still dramatically improve the number of small weight error patterns that can be corrected, therefore potentially increasing the guaranteed error correction capability of a given code.

Note that in the definition of a decoder diversity set, we do not make any a priori assumptions on the cardinalities of each correctable subset $\mathcal{E}_{\mathrm{D}_{i}}$. Typically, strong decoders have large correctable subsets $\mathcal{E}_{\mathrm{D}_{i}}$, while other decoders which are selected to correct very specific error patterns could have a small correctable subset. There are different ways to compose the diversity set $\mathcal{D}$ from $\mathrm{D}_{i}$ 's in order to cover the set $\mathcal{E}$ with the sets $\mathcal{E}_{\mathrm{D}_{i}}$. Two distinct ways are illustrated in Fig. 1. Fig. 1 (a) shows a case where the set of error events $\mathcal{E}$ (represented as a big square) is paved with nearly equally powerful decoders (smaller overlapping squares of similar sizes). Fig. 1(b) shows another type of covering corresponding to using one strong decoder and a number of weaker decoders (smaller rectangles) dedicated to "surgical" correction of specific error patterns not correctable by the strong decoder.

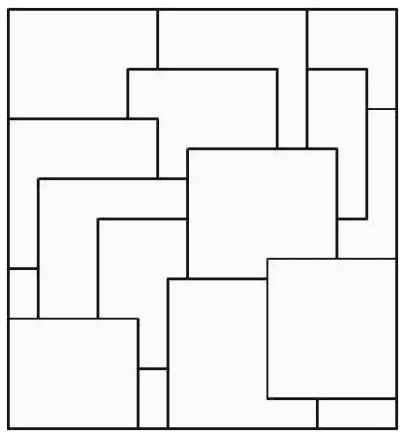

(a)

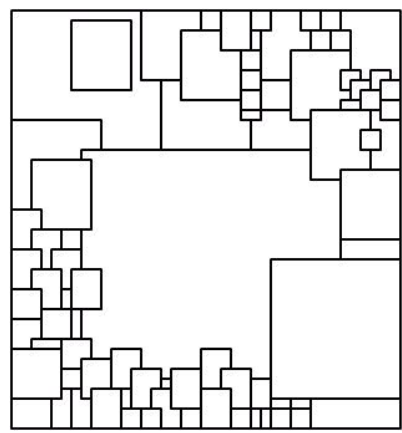

(b)
Figure 1. Typical ways in which decoder diversity can correct all error patterns from a pre-determined set $\mathcal{E}$.

\section{B. Error Sets}

As mentioned previously, our main goal is to find a, possibly small, decoder diversity set $\mathcal{D}$ which guarantees correction of a fixed number of errors $t$. In this section, we describe the error sets that will be used for the selection of FAIDs in $\mathcal{D}$.

Let $G^{\prime}$ be a subgraph that is present in the Tanner graph $G$ of code $\mathcal{C}$. $G^{\prime}$ defines typically closed topological structures such as trapping sets. Let $\mathcal{E}^{k}\left(G^{\prime}\right)$ denote the set of all error patterns of weight $k$ whose support lies entirely in the variable node set of subgraph $G^{\prime}$ :

$$
\mathcal{E}^{k}\left(G^{\prime}\right)=\left\{\mathbf{e}: w(\mathbf{e})=k, \operatorname{supp}(\mathbf{e}) \subseteq V^{\prime}\right\} .
$$

Note that $\mathcal{E}^{k}(G)$ denotes the set of all $k$-error patterns in the code $\mathcal{C}$. For simplicity, we shall denote this particular set as $\mathcal{E}^{k}$ instead of $\mathcal{E}^{k}(G)$. Also let $\mathcal{E}^{[t]}=\bigcup_{k=1}^{t} \mathcal{E}^{k}$ denote the set of all error patterns whose weight is at most $t$.

A brute force approach to ensure a $t$-guaranteed error correction capability is to consider all the error patterns in the set $\mathcal{E}^{[t]}$ for the design of the diversity set $\mathcal{D}$. Obviously, the cardinality of such an error pattern set is too large for a practical analysis. Instead, we shall consider smaller error pattern sets, based on the knowledge of the trapping set distribution of the code $\mathcal{C}$. It is reasonable to assume that the errors patterns that are the most difficult to correct for the iterative decoders are patterns whose support is concentrated in the topological neighborhood of trapping sets.

Recall that $\left\{\mathbf{T}_{i, \mathcal{T}} \mid i=1, \ldots, N_{\mathcal{T}}\right\}$ denotes the collection of all $(a, b)$ trapping sets of type $\mathcal{T}$ that are present in code $\mathcal{C}$. Let $\mathcal{E}^{k}(\mathcal{T})$ denote the set of error patterns of weight $k$ whose support lies in a $(a, b)$ trapping set $\mathbf{T}_{\mathbf{i}, \mathcal{T}}$ of type $\mathcal{T}$. More precisely,

$$
\mathcal{E}^{k}(\mathcal{T})=\left\{\mathbf{e}: w(\mathbf{e})=k, \operatorname{supp}(\mathbf{e}) \subseteq \mathbf{T}_{i, \mathcal{T}} \quad i \in\left\{1, \ldots, N_{\mathcal{T}}\right\}\right\}
$$

The cardinality of $\mathcal{E}^{k}(\mathcal{T})$ is given by $\left|\mathcal{E}^{k}(\mathcal{T})\right|=\left(\begin{array}{l}a \\ k\end{array}\right) N_{\mathcal{T}}$.

Now, let $\Lambda_{a, b}$ denotes the set of all trapping sets of different types present in the code $\mathcal{C}$ that have the same parameters $(a, b)$. The error sets $\mathcal{E}^{k}\left(\Lambda_{a, b}\right)$ and $\mathcal{E}^{[t]}\left(\Lambda_{a, b}\right)$ associated with $\Lambda_{a, b}$ are defined as follows:

$$
\mathcal{E}^{k}\left(\Lambda_{a, b}\right)=\bigcup_{\mathcal{T} \in \Lambda_{a, b}} \mathcal{E}^{k}(\mathcal{T}) \quad \mathcal{E}^{[t]}\left(\Lambda_{a, b}\right)=\bigcup_{k=1}^{t} \mathcal{E}^{k}\left(\Lambda_{a, b}\right)
$$

Finally, $\Lambda^{\{A, B\}}$ is the set containing all $(a, b)$ trapping sets of different types for different values of $a \leq A$ and $b \leq B$, i.e. $\Lambda^{(A, B)}=\bigcup_{0 \leq a \leq A, 0 \leq b \leq B} \Lambda_{a, b}$ and its associated error set is:

$$
\mathcal{E}^{[t]}\left(\Lambda^{(A, B)}\right)=\bigcup_{0 \leq a \leq A, 0 \leq b \leq B} \mathcal{E}^{[t]}\left(\Lambda_{a, b}\right)
$$

Note that $\mathbf{T}_{i, \mathcal{T}} \subseteq \mathcal{T} \subseteq \Lambda_{a, b} \subseteq \Lambda_{A, B}$. Similarly, $\mathcal{E}^{k}(\mathcal{T}) \subseteq$ $\mathcal{E}^{k}\left(\Lambda_{a, b}\right) \subseteq \mathcal{E}^{[t]}\left(\Lambda_{a, b}\right) \subseteq \mathcal{E}^{[t]}\left(\Lambda^{(A, B)}\right) \subseteq \mathcal{E}^{[t]}$. Clearly, the cardinality of $\mathcal{E}^{[t]}\left(\Lambda^{(A, B)}\right)$ is much lower than $\mathcal{E}^{[t]}$, and it can be further reduced by taking into account certain structural properties that the Tanner graph of the code may have due to a specific LDPC code design. Quasi-cyclic codes are prime examples of structured codes [17]. Tanner graphs of such codes possess many trapping sets that are not only isomorphic in the sense of their topological structure, but also have identical neighborhoods. Therefore it suffices to consider error patterns associated with any one of these isomorphic topologies rather than considering all of them. Certain LDPC code constructions 
can ensure that the codes have even more structural properties than just the quasi-cyclicity. A notable example of constrained algebraic construction is reported in [17], in which the existence of three types of group automorphisms reduces the number of trapping sets of maximum size $(A, B)$ that need to be considered by several orders of magnitude. More details on the example of the $(155,64)$ Tanner code shall be provided in Section IV and appendix A.

From the standpoint of computational complexity, it is indeed important to limit the maximum size of the trapping sets that are included in the set $\Lambda^{(A, B)}$. We now provide a conjecture that gives a criterion for the choice of the values of $A$ and $B$, which are needed for defining the error sets.

Conjecture 1. If there exists a decoder diversity set $\mathcal{D}$ that corrects all patterns in the set $\mathcal{E}^{[t]}\left(\Lambda^{(A, B)}\right)$ on the code $\mathcal{C}$ with $A=2 t$ and sufficiently large $B$, then the decoder diversity set $\mathcal{D}$ will also correct all error patterns up to weight $t$ on the code $\mathcal{C}$ with high probability.

This conjecture was found to be valid for the test cases that we have analyzed. The first remark concerns the choice of $B$. Typically it has been observed that, in the case of columnweight $d_{v}=3$ LDPC codes, most harmful $(a, b)$ trapping sets have small values of $b$. Note that this is not the case anymore for LDPC codes with $d_{v}=4$, as explained with the concept of absorbing sets in [18].

The above conjecture is analogous to the condition for correcting $t$ errors by the MLD, which requires the Hamming weight of error patterns to be lower than $\left\lfloor d_{\text {min }} / 2\right\rfloor$. In other words, if a decoder $\mathrm{D}_{i} \in \mathcal{D}$ cannot correct all weight- $t$ error patterns whose support is entirely contained on trapping sets of size smaller than $2 t$, then it is more likely to not be able to correct more scattered weight- $t$ error patterns as well: topologically concentrated error patterns are more difficult to correct.

At the present stage of this work, we have not found any counter-example, but have not been able to prove the conjecture. We have analyzed several codes, and for this paper, we present the results of the $(155,64)$ Tanner code for which the conjecture was verified.

Based on the above conjecture, we now see that considering the set $\mathcal{E}^{[t]}\left(\Lambda^{(A, B)}\right)$ instead of $\mathcal{E}^{[t]}$ is argued to be sufficient for determining the decoder diversity set that ensures guaranteed error correction capability of $t$ with a high probability, and this has a significant complexity reduction, as will be shown on the $(155,64)$ Tanner code.

\section{Generation of FAID Diversity Sets}

We now present the procedure for obtaining the FAID diversity set that guarantees the correction of all error patterns in the set $\mathcal{E}^{[t]}\left(\Lambda^{(A, B)}\right)$. We shall denote this set by $\mathcal{D}^{[t]}$.

Let us assume that we are given a large set of candidate FAIDs $\mathcal{D}_{\text {base }}$ that are considered for possible inclusion in the diversity set. This set could be obtained from simulations on different codes or by using a selection technique that was presented in [8]. Our goal is to build a possibly small set $\mathcal{D}^{[t]}$ from FAIDs belonging to $\mathcal{D}_{\text {base }}$, that collectively corrects all error patterns in $\mathcal{E}^{[t]}\left(\Lambda^{(A, B)}\right)$. In essence, the procedure described in Algorithm 1 runs over all error patterns in $\mathcal{E}^{[t]}\left(\Lambda^{(A, B)}\right)$ and determines their correctability when decoded by different FAIDs from $\mathcal{D}_{\text {base. }}$. In Algorithm $1, N_{I}$ is the maximum number of decoding iterations and $\mathcal{E}_{\mathrm{D}_{i}}^{r}$ denotes the subset of error patterns of $\mathcal{E}^{r}$ that are correctable by the FAID $\mathrm{D}_{i}$.

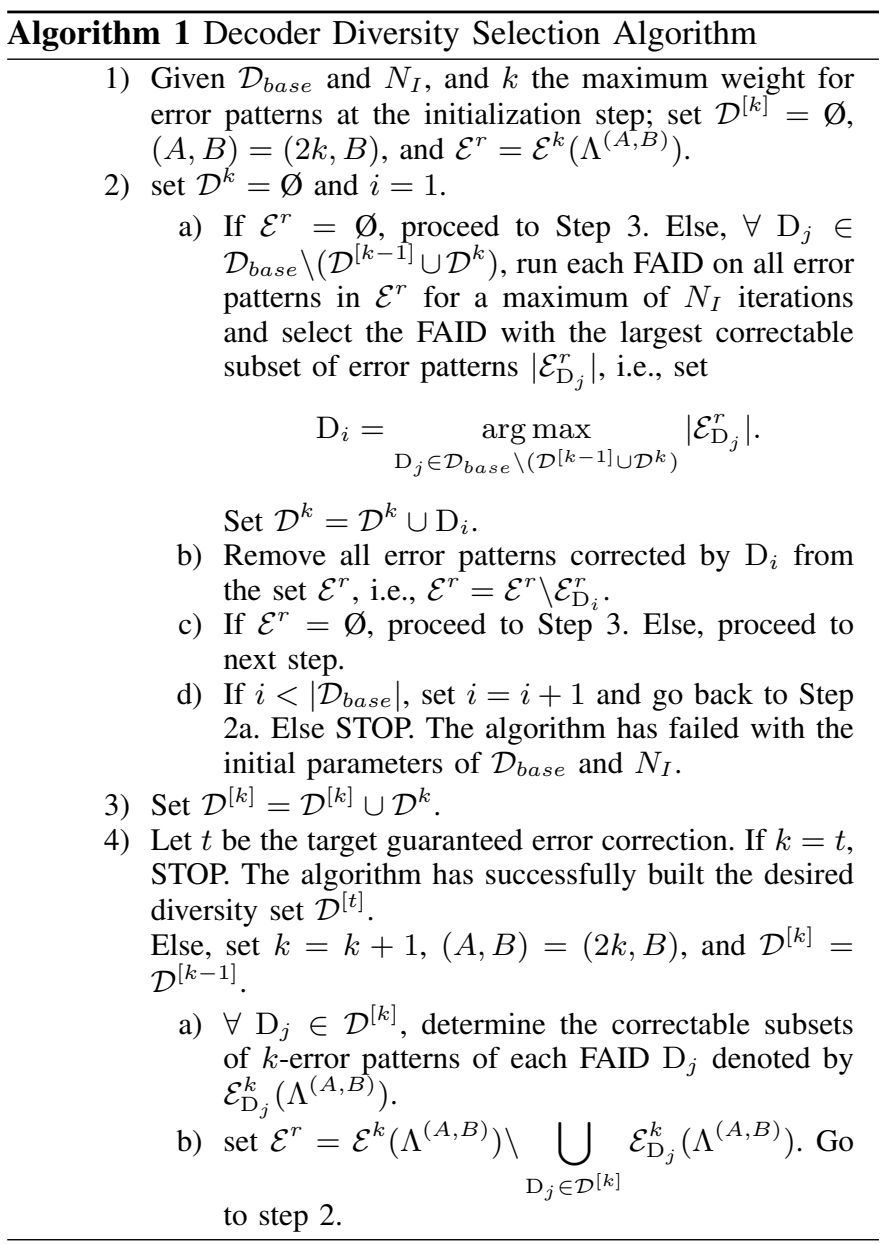

The algorithm starts by building the diversity set $\mathcal{D}^{[k]}$ for a given $k$, then iteratively expands to the diversity sets $\mathcal{D}^{[k+1]}, \mathcal{D}^{[k+2]}, \ldots, \mathcal{D}^{[t]}$ by including more and more FAIDs from $\mathcal{D}_{\text {base }}$ that collectively correct error patterns with increasing weight in $\mathcal{E}^{[t]}\left(\Lambda^{(A, B)}\right)$. The iterative selection of FAIDs is carried out by keeping track, at each iterative stage, of the set of unresolved error patterns $\mathcal{E}^{r} \subset \mathcal{E}^{[t]}\left(\Lambda^{(A, B)}\right)$ which are not collectively correctable by the FAIDs selected so far, and then choosing additional FAIDs to correct these patterns. For example, if $\mathrm{D}_{1}, \mathrm{D}_{2}, \ldots, \mathrm{D}_{L}$ are the FAIDs selected so far for $\mathcal{D}^{[t]}$, and $\mathcal{E}_{\mathrm{D}_{i}}^{[t]}$ denotes the subset of error patterns correctable by FAID $\mathrm{D}_{i}$, then the set of unresolved error patterns is

$$
\mathcal{E}^{r}=\mathcal{E}^{[t]}\left(\Lambda^{(A, B)}\right) \backslash \bigcup_{1 \leq i \leq L} \mathcal{E}_{\mathrm{D}_{i}}^{[t]} .
$$

The algorithm terminates when $\mathcal{E}^{r}=\varnothing$, which means that the set of FAIDs selected up to that point collectively correct all error patterns in $\mathcal{E}^{[t]}\left(\Lambda^{(A, B)}\right)$, and therefore constitute the desired diversity set $\mathcal{D}^{[t]}$. Assuming that Conjecture 
1 holds, the obtained diversity set $\mathcal{D}^{[t]}$ will guarantee a correction of $t$ errors on the LDPC code $\mathcal{C}$. As a side result, the algorithm also gives the FAID diversity sets $\mathcal{D}^{[k]}$ for $k<t$.

For example, suppose we want to build a decoder diversity set $\mathcal{D}^{[7]}$ that achieves a guaranteed error correction of $t=7$ on a code $\mathcal{C}$, and suppose we know that all FAIDs in the given $\mathcal{D}_{\text {base }}$ guarantee a correction of $t=4$. We then choose an intitial value of $k=5$ in Step 1 of the algorithm. The algorithm then starts by building the decoder diversity set $\mathcal{D}^{[5]}$ on the considered error set $\mathcal{E}^{5}\left(\Lambda^{(10, B)}\right)$ with the given choices of $\mathcal{D}_{\text {base }}, N_{I}$, and $B$. The FAIDs are selected from $\mathcal{D}_{\text {base }}$ in a greedy manner and included in $\mathcal{D}^{[5]}$ until all error patterns in $\mathcal{E}^{5}\left(\Lambda^{(10, B)}\right)$ are collectively corrected. Then the algorithm next considers the error set $\mathcal{E}^{6}\left(\Lambda^{(12, B)}\right)$ in order to build the set $\mathcal{D}^{[6]}$. First, all the error patterns correctable by the set $\mathcal{D}^{[5]}$ are removed from the set $\mathcal{E}^{6}\left(\Lambda^{(12, B)}\right)$ to constitute the set $\mathcal{E}^{r}$. Then additional FAIDs from $\mathcal{D}_{\text {base }}$ are selected to correct all the error patterns remaining in $\mathcal{E}^{r}$, which, together with the FAIDs in $\mathcal{D}^{[5]}$, forms the diversity set $\mathcal{D}^{[6]}=\mathcal{D}^{[5]} \cup \mathcal{D}^{6}$. The algorithm repeats the procedure for building $\mathcal{D}^{[7]}$ by operating on the set of error patterns in $\mathcal{E}^{7}\left(\Lambda^{(14, B)}\right)$.

Note that the choices of $N_{I}$ and $\mathcal{D}_{\text {base }}$ can play an important role inn whether or not the algorithm is successful in building the desired decoder diversity set. Determining the optimal $N_{I}$ is beyond the scope of this paper. However, if the algorithm fails in Step 2d, then increasing the value of $N_{I}$ or considering a larger set for $\mathcal{D}_{\text {base }}$ typically allows the algorithm to progress further. We adopted this strategy to obtain a decoder diversity set ensuring a 7 -guaranteed error correction on the $(155,64)$ Tanner code, as shown in the next section.

\section{CASE STUdy: GuARANTEED ERROR CORRECTION ON THE $(155,64)$ TANNER CODE}

We shall now use the $(155,64)$ Tanner code [12], [17], as an example to illustrate how the concept of decoder diversity can be used to increase the guaranteed error-correction capability of the code with reasonable complexity. The $(155,64)$ Tanner code, which is an LDPC code with regular column weight $d_{v}=3$ and row weight $d_{c}=5$, is a particularly good test case for the following reasons. First, the difference between its minimum distance $d_{\min }=20$ and its minimum pseudodistance $w_{p}^{\text {min }} \simeq 10$ is large [19], which means that the difference in the guaranteed error correction capability between traditional iterative decoders (Gallager-B, Min-Sum, BP) and the MLD is expected to be large. Therefore, there is a scope for improvement in reducing this gap using the approach of FAID decoder diversity. Second, the $(155,64)$ Tanner code is sufficiently small and structured (the code has quasi-cyclicity equal to 31) so that a brute force checking of whether all error patterns up to a certain weight- $t$ are corrected by a decoder diversity set can be carried out by Monte Carlo simulations with reasonable computation time.

For comparisons, Table II shows the $t$-guaranteed error correction capability of the existing decoders on the Tanner code. We also found by exhaustively checking through simulations [11] that there is no 7-level FAID that can guarantee a correction of $t>5$ on this particular code. However, using the approach of decoder diversity, we show that it is possible to increase the guaranteed error correction capability of the code to $t=7$. As mentioned in the previous section, we only consider error patterns belonging to $\mathcal{E}^{[t]}\left(\Lambda^{(A, B)}\right)$ where $A=2 t$ and $B$ is large enough. The question of the optimal value for parameter $B$ remains open. $B$ should be set sufficiently large such that Conjecture 1 is verified, but on the other hand, $B$ should be chosen sufficiently small such that the computational complexity of the decoder selection is not too heavy. For $d_{v}=3 \mathrm{LDPC}$ codes and this Tanner code in particular, it appeared from our experiments that $B=3$ was not sufficient for Conjecture 1 to be valid. To be explicit, we found a weight-7 error pattern that was not corrected by the diversity set of FAIDs we selected to collectively correct all weight- 7 error patterns located in TSs with $B=3$. However, a value of $B=4$ was sufficient for all our test cases.

Table II

$t$-GUARANTEED ERROR CORRECTION CAPABILITY OF DIFFERENT DECODERS ON THE $(155,64)$ TANNER CODE

\begin{tabular}{|c|r|r|}
\hline$t$ & Algorithm & Reference \\
\hline 3 & Gallager A and Gallager B & {$[7]$} \\
\hline 4 & Min-Sum and Belief Propagation & {$[10]$} \\
\hline 5 & 5-level and 7-level FAIDs & {$[13]$} \\
\hline
\end{tabular}

Table III

$t$-GUARANTEED ERROR CORRECTION CAPABILITY OF FAID DIVERSITY WITH INCREASING NUMBER OF DECODERS AND ITERATIONS

\begin{tabular}{|l|l|l|}
\hline$t$ & $N_{\mathcal{D}}$ & $N_{I}$ \\
\hline 5 & 1 & 15 \\
\hline 6 & 9 & 50 \\
\hline 7 & 243 & 120 \\
\hline
\end{tabular}

The graph structure of this Tanner code satisfies additional properties on top of the quasi-cyclicity property [12]. Tanner proposed a code construction based on subgroups of $\operatorname{GF}(q)$. The classical additive shift group gives rise to the quasicyclic form of the code, while two multiplicative subgroups associated with rows and columns of the parity-check matrix induces additional group automorphisms on the graph. These isomorphic transformations allow for further reduction in the number of error patterns that need to be considered. Following notations of [12], the transformations $\sigma, \pi$, and $\rho$ act on the indices of the variable nodes and preserve the topological structures. The transformation $\sigma$ comes from the quasi-cyclicity of the code and allows then a constant reduction factor of $L=31$ for all the TS topologies, while the other transformations $\pi$ and $\rho$ can bring another factor of reduction, depending on the type and location of the TS. More details on the three different transformations that the Tanner graph of this code follows are reported in Appendix A.

The full enumeration of trapping sets with $a \leq 14$ and $b \leq 4$ is presented in Table IV. The first column of the table gives the $(a, b)$ parameters, and the second column indicates the TS 
Table IV

TRAPPING SET SPECTRUM OF THE $(155,64)$ TANNER CODE

\begin{tabular}{|c|c|c|c|c|}
\hline $\mathcal{T}$ & TS-label & $N_{\mathcal{T}}$ & $N_{\sigma(\mathcal{T})}$ & $N_{\sigma(\pi(\rho(\mathcal{T})))}$ \\
\hline$(5,3)$ & $\left(5,3 ; 8^{3}\right)$ & 155 & 15 & 1 \\
\hline$(6,4)$ & $\left(6,4 ; 8^{1} 10^{2}\right)$ & 930 & 30 & 2 \\
\hline$(7,3)$ & $\left(7,3 ; 8^{3} 10^{2} 14^{2}\right)$ & 930 & 30 & 2 \\
\hline$(8,2)$ & $\left(8,2 ; 8^{3} 10^{4} 12^{2} 14^{4} 16^{2}\right)$ & 465 & 15 & 1 \\
\hline \multirow[t]{2}{*}{$(8,4)$} & 4 types & 5012 & 165 & 11 \\
\hline & $\begin{array}{l}\left(8,4 ; 8^{3} 12^{2} 16^{2}\right) \\
\left(8,4 ; 8^{1} 10^{2} 12^{2} 14^{2}\right) \\
\left(8,4 ; 8^{1} 10^{3} 12^{1} 14^{1} 16^{1}\right) \\
\left(8,4 ; 8^{1} 10^{4} 16^{2}\right)\end{array}$ & & $\begin{array}{l}45 \\
15 \\
90 \\
15 \\
\end{array}$ & $\begin{array}{l}3 \\
1 \\
6 \\
1\end{array}$ \\
\hline \multirow[t]{2}{*}{$(9,3)$} & 3 types & 1860 & 60 & 4 \\
\hline & $\begin{array}{l}\left(9,3 ; 8^{1} 10^{4} 12^{4} 14^{2} 16^{2} 18^{2}\right) \\
\left(9,3 ; 8^{1} 10^{5} 12^{2} 14^{2} 16^{4}\right) \\
\left(9,3 ; 8^{3} 10^{2} 12^{2} 14^{4} 16^{2} 18^{2}\right) \\
\end{array}$ & & $\begin{array}{l}15 \\
30 \\
15 \\
\end{array}$ & $\begin{array}{l}1 \\
2 \\
1\end{array}$ \\
\hline \multirow[t]{2}{*}{$(10,2)$} & 2 types & 1395 & 45 & 3 \\
\hline & $\begin{array}{l}\left(10,2 ; 8^{1} 10^{6} 12^{5} 14^{4} 16^{6} 18^{5} 20^{2}\right) \\
\left(10,2 ; 8^{3} 10^{5} 12^{2} 14^{6} 16^{6} 18^{2} 20^{4}\right)\end{array}$ & & $\begin{array}{l}15 \\
30\end{array}$ & $\begin{array}{l}1 \\
2\end{array}$ \\
\hline$(10,4)$ & 27 types & 29295 & 945 & 63 \\
\hline$(11,3)$ & 11 types & 6200 & 200 & 14 \\
\hline \multirow[t]{2}{*}{$(12,2)$} & 2 types & 930 & 30 & 2 \\
\hline & $\begin{array}{l}\left(12,2 ; 8^{1} 10^{6} 12^{6} 14^{6} 16^{9} 18^{9} 20^{8} 22^{7} 24^{4}\right) \\
\left(12,2 ; 8^{4} 10^{2} 12^{4} 14^{4} 16^{8} 18^{1} 220^{6} 22^{8} 24^{2}\right)\end{array}$ & & $\begin{array}{l}15 \\
15 \\
\end{array}$ & $\begin{array}{l}1 \\
1\end{array}$ \\
\hline$(12,4)$ & 170 types & 196440 & 6240 & 416 \\
\hline$(13,3)$ & 53 types & 34634 & 1155 & 79 \\
\hline
\end{tabular}

cycle inventory of different $(a, b)$ TS types (the cycle inventory is omitted for the parameters that allow too many cycleinventory types). The last three columns show the numbers of trapping sets that need to be checked by Algorithm 1 when the code group automorphisms are exploited. $N_{\mathcal{T}}$ corresponds to the number of trapping sets present without taking any code structure into account, $N_{\sigma(\mathcal{T})}$ corresponds to the number of trapping sets present after taking into account the quasi-cyclic property obtained from the transformation $\sigma$, and $N_{\sigma(\pi(\rho(\mathcal{T})))}$ corresponds to the number of trapping sets present after taking all three transformations $\sigma, \pi$ and $\rho$ into consideration.

Table $\mathrm{V}$ shows the structure and number of the lowest weight codewords of different types, which correspond to the smallest TSs with $b=0$.

The trapping sets have been enumerated using the modified impulse algorithm, which is known to be an efficient algorithm to find low-weight codewords or near-codewords of a given short length LDPC code [20], [21]. When the number of types was too large, we did not indicate the details of the TS notation. It is clear from the table that the number of topologies needed to be considered to characterize the behavior of an iterative decoder on the Tanner code could be greatly reduced. Actually, the number of structures (including isomorphic) of given type $\mathcal{T}$ present in the code could be multiples of either $L d_{c} d_{v}=465, L d_{c}=155$ or $L d_{v}=93$ and this number is reduced for the analysis by the transformations
$\sigma(\pi(\rho(\mathcal{T})))$. The TS of type $\left(5,3 ; 8^{3}\right)$ is an example where there are $L d_{c}=155$ such structures in the Tanner code, while $(20,0)$-type-III codewords is an example where there are $L d_{v}=93$ such structures.

\section{A. Error Sets for the Tanner Code}

The error sets that we have considered for the Tanner code are shown in Table VI along with their cardinalities. The cardinalities of the error sets have been reduced using the structural properties $\sigma, \pi$, and $\rho$ of the Tanner code to:

$$
\left|\mathcal{E}^{k}(\mathcal{T})\right|=\left(\begin{array}{c}
a \\
k
\end{array}\right) N_{\sigma(\pi(\rho(\mathcal{T})))} .
$$

where $N_{\sigma(\pi(\rho(\mathcal{T})))}$ is the value obtained from Table IV.

One can further reduce the number of error patterns considered in each error set, since a particular error pattern belonging to an error set of a small trapping set may also be included in the error set of a larger trapping set containing the smaller one. For example, a 5-error pattern on one of the TS $(9,3)$ could be also listed as one of the 5-error patterns in the TS $(8,2)$ if $(8,2)$ is contained in $(9,3)$. Therefore, we also take this into account by including only the error patterns in the error set $\mathcal{E}^{[k]}\left(\Lambda_{a, b}\right)$ that are distinct from all error patterns in $\mathcal{E}^{[k]}\left(\Lambda_{a^{\prime}, b^{\prime}}\right)$ with $a^{\prime}<a$ and $b^{\prime}<b$. This leads to a further reduction in the number of error patterns considered in error sets, and the final number is reported at the bottom 
Table V

LOW-WEIGHT CODEWORDS SPECTRUM OF THE $(155,64)$ TANNER CODE

\begin{tabular}{|l|l|l|l|l|}
\hline $\mathcal{T}$ & TS-label & $N_{\mathcal{T}}$ & $N_{\sigma(\mathcal{T})}$ & $N_{\sigma(\pi(\rho(\mathcal{T})))}$ \\
\hline \multirow{2}{*}{$(20,0)$} & 3 types & $\mathbf{1 0 2 3}$ & $\mathbf{3 3}$ & $\mathbf{3}$ \\
\cline { 2 - 5 } & type-I & 465 & 15 & 1 \\
& type-II & 465 & 15 & 1 \\
& type-III & 93 & 3 & 1 \\
\hline$(22,0)$ & 14 types & $\mathbf{6 2 0 0}$ & $\mathbf{2 0 0}$ & $\mathbf{1 4}$ \\
\hline$(24,0)$ & 97 types & $\mathbf{4 3 8 6 5}$ & $\mathbf{1 4 1 5}$ & $\mathbf{9 7}$ \\
\hline
\end{tabular}

Table VI

CARDINALITIES OF ERROR SETS CONSIDERED FOR THE $(155,64)$ TANNER CODE. FOR EACH $k$-ERROR PATTERN SET, WE INDICATE THE TOTAL NUMBER OF PATTERNS IN THE FIRST COLUMN (BASED ON EQUATION (9)), THE SECOND COLUMN INDICATES THE FINAL NUMBER OF ERROR PATTERNS, AFTER REMOVING THE ISOMORPHIC ONES.

\begin{tabular}{|c|c|c|c|c|c|c|c|c|}
\hline \multicolumn{3}{|c|}{ 5-errors } & \multirow{2}{*}{\multicolumn{3}{|c|}{ 6-errors }} & & \multirow{3}{*}{\multicolumn{2}{|c|}{ 7-errors }} \\
\hline \multirow{2}{*}{$\begin{array}{l}\mathcal{E}^{[5]}\left(\Lambda_{5,3}\right) \\
\mathcal{E}^{[5]}\left(\Lambda_{6,4}\right)\end{array}$} & \multirow{2}{*}{$\begin{array}{l}1 \\
12\end{array}$} & \multirow{2}{*}{$\begin{array}{l}1 \\
12\end{array}$} & & & & & & \\
\hline & & & $\mathcal{E}^{[6]}\left(\Lambda_{6,4}\right)$ & 2 & 2 & & & \\
\hline $\mathcal{E}^{[5]}\left(\Lambda_{7,3}\right)$ & 42 & 23 & $\mathcal{E}^{[6]}\left(\Lambda_{7,3}\right)$ & 14 & 11 & $\mathcal{E}^{[7]}\left(\Lambda_{7,3}\right)$ & 2 & 2 \\
\hline $\mathcal{E}^{[5]}\left(\Lambda_{8,2}\right)$ & 56 & 20 & $\mathcal{E}^{[6]}\left(\Lambda_{8,2}\right)$ & 28 & 15 & $\mathcal{E}^{[7]}\left(\Lambda_{8,2}\right)$ & 8 & 6 \\
\hline $\mathcal{E}^{[5]}\left(\Lambda_{8,4}\right)$ & 616 & 398 & $\mathcal{E}^{[6]}\left(\Lambda_{8,4}\right)$ & 308 & 240 & $\mathcal{E}^{[7]}\left(\Lambda_{8,4}\right)$ & 88 & 79 \\
\hline $\mathcal{E}^{[5]}\left(\Lambda_{9,3}\right)$ & 504 & 100 & $\mathcal{E}^{[6]}\left(\Lambda_{9,3}\right)$ & 336 & 110 & $\mathcal{E}^{[7]}\left(\Lambda_{9,3}\right)$ & 144 & 72 \\
\hline $\mathcal{E}^{[5]}\left(\Lambda_{10,2}\right)$ & 756 & 399 & $\mathcal{E}^{[6]}\left(\Lambda_{10,2}\right)$ & 630 & 416 & $\mathcal{E}^{[7]}\left(\Lambda_{10,2}\right)$ & 360 & 277 \\
\hline $\mathcal{E}^{[5]}\left(\Lambda_{10,4}\right)$ & 15876 & 7064 & $\mathcal{E}^{[6]}\left(\Lambda_{10,4}\right)$ & 13230 & 7860 & $\mathcal{E}^{[7]}\left(\Lambda_{10,4}\right)$ & 7560 & 5421 \\
\hline & & & $\mathcal{E}^{[6]}\left(\Lambda_{11,3}\right)$ & 6468 & 1958 & $\mathcal{E}^{[7]}\left(\Lambda_{11,3}\right)$ & 4620 & 1894 \\
\hline & & & $\mathcal{E}^{[6]}\left(\Lambda_{12,2}\right)$ & 1848 & 766 & $\mathcal{E}^{[7]}\left(\Lambda_{12,2}\right)$ & 1584 & 857 \\
\hline & & & $\mathcal{E}^{[6]}\left(\Lambda_{12,4}\right)$ & 384384 & 163562 & $\mathcal{E}^{[7]}\left(\Lambda_{12,4}\right)$ & 329472 & 187360 \\
\hline & & & & & & $\mathcal{E}^{[7]}\left(\Lambda_{13,3}\right)$ & 135564 & 31890 \\
\hline & & & & & & $\mathcal{E}^{[7]}\left(\Lambda_{14,2}\right)$ & 37752 & 8157 \\
\hline & & & & & & $\mathcal{E}^{[7]}\left(\Lambda_{14,4}\right)$ & 9129120 & 3326862 \\
\hline
\end{tabular}

\begin{tabular}{|l|l|l|l|l|l|}
\hline \multicolumn{2}{|c|}{ 5-errors } & \multicolumn{2}{|c|}{ 6-errors } & \multicolumn{2}{c|}{ 7-errors } \\
\hline $\mathcal{E}^{[5]}\left(\Lambda^{(10,4)}\right)$ & 8017 & $\mathcal{E}^{[6]}\left(\Lambda^{(12,4)}\right)$ & 174940 & $\mathcal{E}^{[7]}\left(\Lambda^{(14,4)}\right)$ & 3562877 \\
\hline $\mathcal{E}^{[5]}$ & 698526906 & $\mathcal{E}^{[6]}$ & 17463172650 & $\mathcal{E}^{[7]}$ & 371716103550 \\
\hline \multicolumn{2}{|c|}{ Comp. Reduction Factor } & \multicolumn{2}{|c|}{ Comp. Reduction Factor } & \multicolumn{2}{|c|}{ Comp. Reduction Factor } \\
\hline \multicolumn{2}{|c|}{87130} & \multicolumn{2}{|c|}{99824} & \multicolumn{2}{|c|}{104330} \\
\hline
\end{tabular}

of Table VI. From the Table, we can see that the complexity reduction factor in each case is of the order of $10^{5}$, which is very large and in any case sufficient to reduce the complexity of finding the decoder diversity set to a reasonable level. In terms of computational time, using this reduced number of error patterns, it is possible to test one FAID, that is to identify the correctable error patterns in $\mathcal{E}^{[5]}\left(\Lambda^{(10,4)}\right), \mathcal{E}^{[6]}\left(\Lambda^{(12,4)}\right)$ and $\mathcal{E}^{[7]}\left(\Lambda^{(14,4)}\right)$, within only a few minutes. As a result, one can consider a large number of candidate FAIDs in order to optimize the diversity set with Algorithm 1, and starting with a collection of $\left|\mathcal{D}_{\text {base }}\right|=49522$ FAIDs, we were able to identify the diversity sets for the Tanner code presented in the next section in less than 48 hours. Our approach is reproducible for any $d_{v}=3$ LDPC code with short to moderate blocklength, with a roughly constant complexity.

\section{B. Error Correction Results for the Tanner Code}

Let us recall that we consider only 7-level FAIDs for decoder diversity which require only 3 bits of precision for their message representation. Our main results are summarized in Table III. We are able to guarantee a correction of $t=7$ errors on the Tanner code using $N_{\mathcal{D}}=343$ FAIDs with $N_{I}=120$ iterations.

We also verified by brute force Monte Carlo simulations that each of the obtained diversity sets $\mathcal{D}^{[t]}$ for $t=5,6,7$ guarantees a correction of all error patterns of weight at most $t$ on the Tanner code even though only error patterns in $\mathcal{E}^{[t]}\left(\Lambda^{(A, B)}\right)$ with $(A, B)=(2 t, 4)$ were used in the algorithm, thus validating the conjecture stated in Section III-B.

Due to the huge cardinality reduction in the error sets considered (as shown in Table VI), we were able to identify the decoder diversity set for $t=6$ in less than one hour, and for $t=7$ in a few days. Note that decoder diversity does not 
require any post-processing, as it is still an iterative messagepassing decoder with the additional feature that the the variable node update rule $\Phi_{v}$ changes after $N_{I}$ iterations (and the decoder is restarted). Note also that additional complexity reduction can be achieved by exploiting any existing similarities between the update rules of the FAIDs in the decoder diversity set.

In order to illustrate how different 7-level FAIDs in the decoder diversity set can behave in terms of their correctability of different error patterns in the error set, we provide two examples with the help of Table VII.

Table VII

STATISTICS ON THE ERROR CORRECTION OF SEVERAL FAIDS USED IN THE DECODER DIVERSITY SETS.

\begin{tabular}{|c|c|c|c|c|c|c|c|c|c|c|}
\hline Decoder $\mathrm{D}_{i}$ & $\mathrm{D}_{0}$ & $\mathrm{D}_{1}$ & $\mathrm{D}_{2}$ & & $D_{3}$ & $\mathrm{D}_{4}$ & $\mathrm{D}_{5}$ & $\mathrm{D}_{6}$ & $\mathrm{D}_{7}$ & $\mathrm{D}_{8}$ \\
\hline $\mid \mathcal{E}_{\mathbb{D}_{i}}^{[6]}\left(\Lambda^{(11,4)}\right)$ & 10469 & 10560 & 1057 & & 5575 & 10527 & 10488 & 10444 & 10247 & 10454 \\
\hline \multirow[t]{2}{*}{ remaining errors } & 143 & 25 & 12 & & 8 & 5 & 3 & 2 & 1 & 0 \\
\hline & ecoder I & $\mathrm{D}$ & & 10 & $D_{11}$ & $\mathrm{D}_{12}$ & $\mathrm{D}_{13}$ & $\mathrm{D}_{14}$ & $D_{15}$ & $\mathrm{D}_{16}$ \\
\hline \multicolumn{2}{|c|}{$\mathcal{E}_{\mathcal{D}_{i}}^{[7]}\left(\Lambda_{7,3}\right) \cup \mathcal{E}_{\mathcal{D}_{i}}^{[7]}\left(\Lambda_{8,2}\right)$} & 1 & & 1 & 1 & 1 & 1 & 1 & 1 & 1 \\
\hline & Not & 973 & & 103 & 80 & 198 & 9216 & 3450 & 156 & 304 \\
\hline
\end{tabular}

The first part of Table VII shows an example of using equally powerful decoders in the diversity set. Statistics are provided on the number of correctable error patterns $\left|\mathcal{E}_{\mathrm{D}_{i}}^{[6]}\left(\Lambda^{(12,4)}\right)\right|$ by each 7-level FAID in the decoder diversity set $\mathcal{D}^{[6]}$ from the error set $\mathcal{E}^{[6]}\left(\Lambda^{(11,4)}\right)$. The LUT maps of $\Phi_{v}$ that define these 7-level FAIDs are reported in Table VIII of Appendix B. For convenience, we have noted $\mathcal{D}^{[5]}=\left\{\mathbf{D}_{0}\right\}$ and $\mathcal{D}^{[6]}=\mathcal{D}^{[5]} \cup\left\{\mathbf{D}_{1}, \ldots, \mathbf{D}_{8}\right\}$. The total number of error patterns in $\mathcal{E}^{[6]}\left(\Lambda^{(11,4)}\right)$ is $\left|\mathcal{E}^{[6]}\left(\Lambda^{(11,4)}\right)\right|=10612$, which corresponds to all the 6 -error patterns in TS with $a \leq 11$ and $b \leq 4$. From Table VII, we can see that all decoders in $\mathcal{D}^{[6]}$ are in fact almost equally powerful in terms of the number of error patterns they correct. However, all 9 decoders when used in combination are able to collectively guarantee an error correction of $t=6$.

The second part of Table VII provides an example of how certain decoders, that we refer to as "surgeon" decoders, can be used to specifically correct certain error patterns not correctable by the particularly good decoders. The statistics shown in the table are for eight different FAIDs (labeled $\mathrm{D}_{9}$ to $\mathrm{D}_{16}$ for convenience) that were selected to correct eight particular 7 -error patterns in the error set $\mathcal{E}^{[7]}\left(\Lambda_{7,3}\right) \cup \mathcal{E}^{[7]}\left(\Lambda_{8,2}\right)$. These eight different FAIDs are required to separately correct each of these error patterns. Moreover, in comparison with the statistics obtained from the FAIDs belonging to $\mathcal{D}^{[6]}$ on the 6-error patterns, these decoders are not as strong as the first nine decoders $\mathbf{D}_{0}$ to $\mathbf{D}_{8}$. Five of them especially have very poor behaviors on the 6-error events.

These surgeon decoders, can be seen as tuned to a particular error event, and are not necessarily included in the set of candidate FAIDs $\mathcal{D}_{\text {base }}$ used for the diversity set selection algorithm. In particular, $\left(\mathrm{D}_{10}, \mathrm{D}_{11}, \mathrm{D}_{12}, \mathrm{D}_{15}, \mathrm{D}_{16}\right)$ were not found in the $\left|\mathcal{D}_{\text {base }}\right|=49522$ FAIDs which were used in the initialization of Algorithm 1. To find these decoders, we increased the number of candidate FAIDs, and looked for the ones which correct the remaining five 7-error patterns.

These two examples clearly show that in order to guarantee $t$ error correction, the decoder diversity sets can pave the space of error sets in very different manners. In summary, for $t=6$ error correction, the decoder diversity set behavior is roughly like that depicted in Fig. 1(a), while for $t=7$ error correction, the decoder diversity set behavior is more like that shown in Fig. 1(b) using both powerful and surgeon decoders. The list of FAIDs $\mathbf{D}_{0}$ to $\mathbf{D}_{8}$ and $\mathbf{D}_{10}$ to $\mathbf{D}_{17}$ are reported in Appendix B.

Fig. 2 shows the remaining 7 -error patterns in $\mathcal{E}^{[7]}$ after the sequential use of the FAIDs in $\mathcal{D}^{[5]}$ followed by the FAIDs in $\mathcal{D}^{[6]} \backslash \mathcal{D}^{[5]}$, and then followed by FAIDs in $\mathcal{D}^{[7]} \backslash \mathcal{D}^{[6]}$.

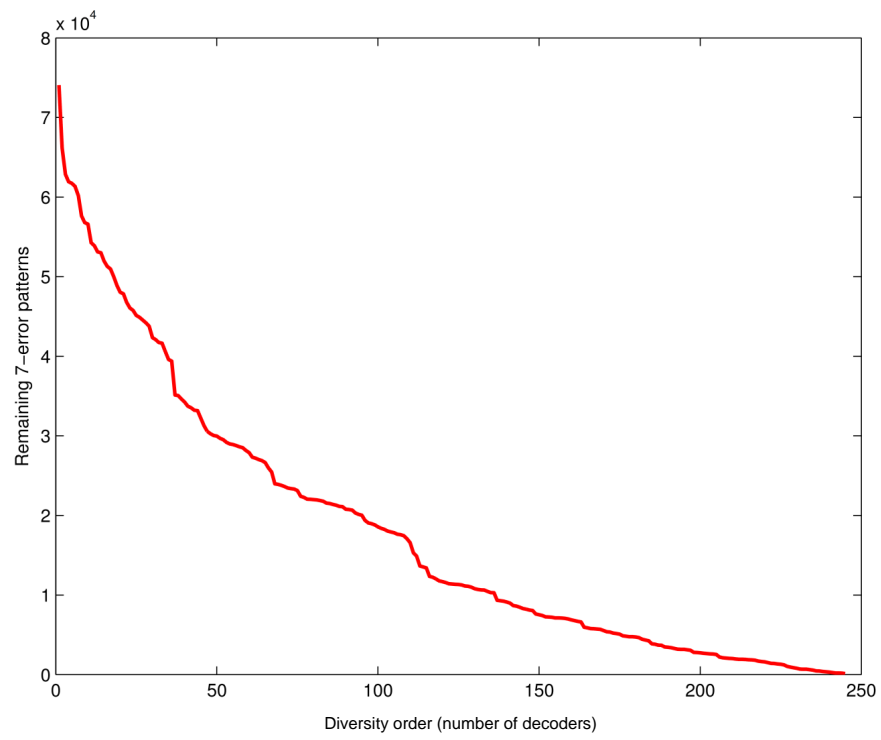

Figure 2. Number of remaining uncorrected 7 -error patterns with sequential use of FAIDs in the diversity sets.

Fig. 3 shows the FER performance of the decoder diversity set $\mathcal{D}^{[7]}$, when simulated on the Tanner code over the BSC channel with cross-over error probability $\alpha$ and with a maximum of $N_{I}=120$ decoding iterations for each decoder. The following sequential schedule is employed for the use of several FAIDs. The order in which the FAIDs are used sequentially is fixed by the identification process of Algorithm 1. For each and every channel noise realization, the FAIDs are run iteratively. When a given FAID $\mathrm{D}_{d} \in \mathcal{D}^{[7]}$ fails to converge after $N_{I}$ iterations, then the channel values are used to reinitialize the next decoder $\mathrm{D}_{d+1}$, until a codeword is found or the maximum number of FAIDs in $\mathcal{D}^{[7]}$ has been reached. Note that in the case $\mathrm{D}_{d}$ converges to a wrong codeword, we declare it as an undetected error, and do not switch to the next decoder. This latter situation never appeared for our simulations on the Tanner code. Since the FAIDs are used sequentially, the average computational time for the Monte-Carlo simulations is roughly the same as if one were using a single FAID. The subsequent FAIDs in the diversity set are triggered only when the previous one fails to converge, and since in the error floor, the first FAID has already a FER $<10^{-7}$, the diversity set is used only at the same rate, which adds a negligible simulation 
time. While finding an efficient hardware implementation for decoder diversity with FAIDs is an important issue to consider, it is one that lies beyond the scope of this paper.

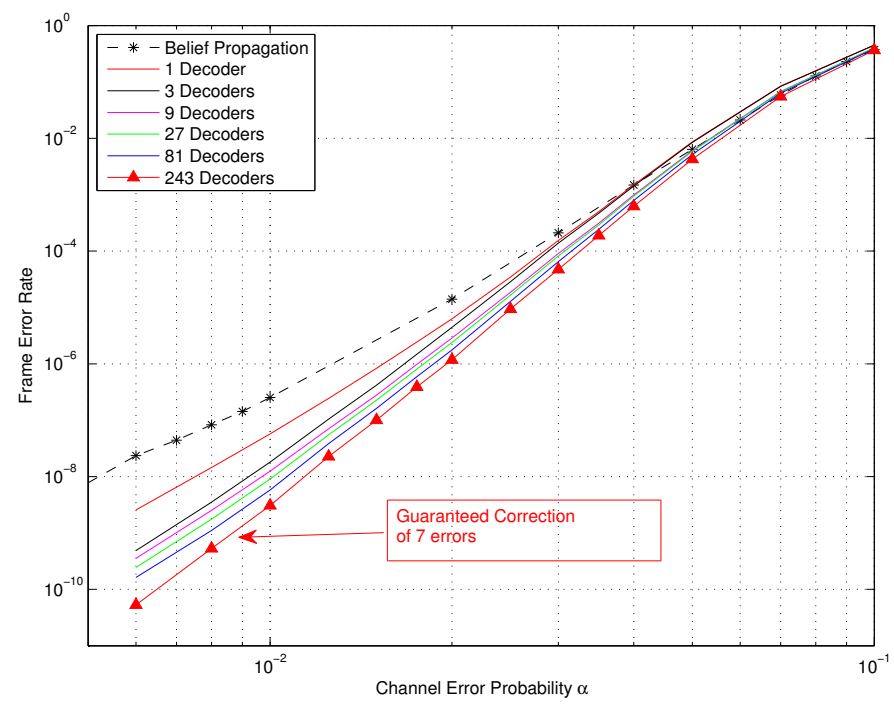

Figure 3. FER results on the Tanner Code with guaranteed error correction of 7 errors.

One can see that, especially in the error floor region, the use of an increasing number of FAIDs increases the slope of the FER curve, and eventually reaches a slope of $t=8$, which corresponds to the minimum weight error pattern that is not corrected by our decoder diversity set $\mathcal{D}^{[7]}$.

\section{CONCLUSIONS}

We introduced a general decoding scheme that utilizes a collection of several different FAIDs, which is referred to as a decoder diversity set, in order to further increase the guaranteed error correction capability of a given LDPC code on the BSC from what is achievable by a single FAID. We provided a methodology to build the decoder diversity sets based on using the trapping set distribution of the code, and considering error patterns that are only associated with the trapping sets present in the code. Using the $(155,64)$ Tanner code as an example, we showed that the structural properties of the code can be exploited to reduce the complexity in terms of reducing the number of considered error patterns by several orders. We were able to increase the guaranteed error correction capability of the $(155,64)$ Tanner code using our approach of decoder diversity, from $t=5$ errors that is achievable by a single FAID to $t=7$ errors by FAID decoder diversity. Note that the BP algorithm is able to guarantee a correction of only $t=4$ on the Tanner code. Also although our discussion throughout this paper primarily focused on the particular example of the Tanner code, the technique can be applied to other codes with regular column weight $d_{v}$, provided that the trapping set distribution is known (which is a reasonable assumption for short to moderate codeword lengths).

Finally, we remark that while the approach proposed in this paper is restricted to the case of the BSC and deals with only hard-decision errors, extensions of this approach to other channels such as the additive white Gaussian channel (AWGNC) is highly non-trivial as the notion of guaranteed error correction only makes sense for hard-decision decoding. However, we believe that the central idea of decoder diversity can still be beneficial for improving the error floor performance over the AWGNC, and extending this idea for the AWGNC is a topic for future research.

\section{APPENDIX A \\ TOPOLOGIES OF THE TANNER CODE}

As explained in [12], there are three types of group automorphisms which preserve the topological structures in the graph of the Tanner code, due to the fact that Tanner's design of the parity-check matrix is based on an array of $\left(d_{v}, d_{c}\right)$ circulants of size $L$, and that the values of shifts for the circulant matrices are chosen from two multiplicative subgroups of the Galois field $\operatorname{GF}(L)$. For ease of understanding, we shall instead present the group automorphisms as simple transformations acting on the indices of the variable nodes in the code. Let $\gamma$ (respectively $\beta$ ) be two elements of $\operatorname{GF}(L)$ with multiplicative order $d_{c}$ (respectively $d_{v}$ ). The parity check matrix is defined by an array of circulants with shift orders $\left\{\gamma^{t} \beta^{r}\right\}_{0 \leq t \leq d_{c}-1,0 \leq r \leq d_{v}-1}$. Now, let the index of a variable node $v_{i}$ be expressed as $i=k * L+l$. We now define the three following transformations acting on the indices of $\mathbf{T}$ that preserve the topology as well as the neighborhood of the TS.

- block-cyclicity: Let $\sigma: V \times\{0, \ldots, L-1\} \rightarrow V$. Then $\sigma\left(v_{i}, t\right)=v_{j} \quad$ where $j=(k * L)+(l+t \bmod (L))$

- row-wise automorphism: Let $\pi: V \times\left\{0, \ldots, d_{c}-1\right\} \rightarrow$ $V$. Then

$$
\begin{aligned}
\pi\left(v_{i}, t\right)=v_{j} \text { where } j= & \left(\left(k+t \bmod \left(d_{c}\right)\right) * L\right) \\
& +\left(\gamma^{t} l \bmod (L)\right)
\end{aligned}
$$

- column-wise automorphism: Let $\rho: V \times\left\{0, \ldots, d_{v}-\right.$ $1\} \rightarrow V$. Then

$$
\rho\left(v_{i}, t\right)=v_{j} \quad \text { where } j=(k * L)+\left(\beta^{t} l \bmod (L)\right)
$$

Consider a TS of size $a$ bits denoted by $\mathbf{T}=$ $\left\{v_{n_{1}}, \ldots, v_{n_{a}}\right\}$. By applying the transformation $\sigma$ on $\mathbf{T}$ such that $\sigma(\mathbf{T}, t)=\left\{\sigma\left(v_{n_{1}}, t\right), \ldots, \sigma\left(v_{n_{a}}, t\right)\right\}$ where $t \in$ $\{0, \ldots, L-1\}$, the induced subgraphs of $\sigma(\mathbf{T}, t)$ and $\mathbf{T}$ are isomorphic to each other in the code $\forall t \in\{0, \ldots, L-1\}$, i.e., they have exactly the same topology and neighborhood. This implies that one has to only consider error patterns associated with one of the isomorphic structures instead of all of them. The same applies for the transformations $\pi$ and $\rho$. By applying all three transformations $\sigma(\pi(\rho(\mathbf{T})))$, the number of trapping sets of a certain type $\mathcal{T}$ that need to be considered is significantly reduced.

\section{APPENDIX B \\ LIST OF 7-LEVEL FAIDS USED IN THE FAID DIVERSITY ALGORITHM}

In Table VIII, we list some of the 7-level FAIDs used in this paper, and which were used in the FAID diversity sets 
Table VIII

LIST OF SOME 7-LEVEL FAIDS USED IN THIS PAPER. THE FIRST NINE FAIDS GUARANTEE AN ERROR CORRECTION OF $t=6$ ON THE $(155,64)$ TANNER CODE.

\begin{tabular}{|c|c|c|c|c|c|c|c|c|c|c|c|c|c|c|c|c|c|c|c|c|c|c|c|c|c|c|c|c|}
\hline FAID & $l_{1,1}$ & $l_{1,2}$ & $l_{1,3}$ & $l_{1,4}$ & $l_{1,5}$ & $l_{1,6}$ & $l_{1,7}$ & $l_{2,2}$ & $l_{2,3}$ & $l_{2,4}$ & $l_{2,5}$ & $l_{2,6}$ & $l_{2,7}$ & $l_{3,3}$ & $l_{3,4}$ & $l_{3,5}$ & $l_{3,6}$ & $l_{3,7}$ & $l_{4,4}$ & $l_{4,5}$ & $l_{4,6}$ & $l_{4,7}$ & $l_{5,5}$ & $l_{5,6}$ & $l_{5,7}$ & $l_{6,6}$ & $l_{6,7}$ & $l_{7,7}$ \\
\hline $\mathbf{D}_{0}$ & $-L_{3}$ & $-L_{3}$ & $-L_{3}$ & $-L_{3}$ & $-L_{3}$ & $-L_{3}$ & $-L_{1}$ & $-L_{3}$ & $-L_{3}$ & $-L_{3}$ & $-L_{2}$ & $-L_{1}$ & $L_{1}$ & $-L_{2}$ & $-L_{2}$ & $-L_{1}$ & $-L_{1}$ & $L_{1}$ & $-L_{1}$ & 0 & 0 & $L_{1}$ & 0 & $L_{1}$ & $L_{2}$ & $L_{1}$ & $L_{3}$ & $L_{3}$ \\
\hline $\mathbf{D}_{1}$ & $-L_{3}$ & $-L_{3}$ & $-L_{3}$ & $-L_{3}$ & $-L_{3}$ & $-L_{3}$ & 0 & $-L_{3}$ & $-L_{3}$ & $-L_{3}$ & $-L_{2}$ & $-L_{2}$ & $L_{1}$ & $-L_{2}$ & $-L_{1}$ & $-L_{1}$ & 0 & $L_{2}$ & $-L_{1}$ & 0 & 0 & $L_{2}$ & 0 & $L_{1}$ & $L_{2}$ & $L_{1}$ & $L_{3}$ & $L_{3}$ \\
\hline $\mathbf{D}_{2}$ & $-L_{3}$ & $-L_{3}$ & $-L_{3}$ & $-L_{3}$ & $-L_{3}$ & $-L_{3}$ & $-L_{1}$ & $-L_{3}$ & $-L_{3}$ & $-L_{2}$ & $-L_{2}$ & $-L_{2}$ & $L_{1}$ & $-L_{2}$ & $-L_{1}$ & $-L_{1}$ & 0 & $L_{1}$ & $-L_{1}$ & 0 & 0 & $L_{3}$ & 0 & $L_{1}$ & $L_{3}$ & $L_{1}$ & $L_{3}$ & $L_{3}$ \\
\hline $\mathbf{D}_{3}$ & $-L_{3}$ & $-L_{3}$ & $-L_{3}$ & $-L_{3}$ & $-L_{3}$ & $-L_{3}$ & $-L_{1}$ & $-L_{3}$ & $-L_{3}$ & $-L_{2}$ & $-L_{2}$ & $-L_{1}$ & $L_{2}$ & $-L_{2}$ & $-L_{1}$ & $-L_{1}$ & 0 & $L_{2}$ & $-L_{1}$ & 0 & 0 & $L_{2}$ & 0 & $L_{1}$ & $L_{3}$ & $L_{1}$ & $L_{3}$ & $L_{3}$ \\
\hline $\mathbf{D}_{4}$ & $-L_{3}$ & $-L_{3}$ & $-L_{3}$ & $-L_{3}$ & $-L_{3}$ & $-L_{3}$ & $-L_{1}$ & $-L_{3}$ & $-L_{3}$ & $-L_{3}$ & $-L_{1}$ & $-L_{1}$ & $L_{1}$ & $-L_{2}$ & $-L_{2}$ & $-L_{1}$ & $-L_{1}$ & $L_{2}$ & $-L_{1}$ & 0 & 0 & $L_{2}$ & 0 & $L_{1}$ & $L_{2}$ & $L_{1}$ & $L_{2}$ & $L_{3}$ \\
\hline $\mathbf{D}_{5}$ & $-L_{3}$ & $-L_{3}$ & $-L_{3}$ & $-L_{3}$ & $-L_{3}$ & $-L_{3}$ & 0 & $-L_{3}$ & $-L_{3}$ & $-L_{3}$ & $-L_{1}$ & $-L_{1}$ & $L_{1}$ & $-L_{2}$ & $-L_{2}$ & $-L_{1}$ & $-L_{1}$ & $L_{2}$ & $-L_{1}$ & 0 & 0 & $L_{2}$ & 0 & $L_{1}$ & $L_{2}$ & $L_{1}$ & $L_{2}$ & $L_{3}$ \\
\hline $\mathbf{D}_{6}$ & $-L_{3}$ & $-L_{3}$ & $-L_{3}$ & $-L_{3}$ & $-L_{3}$ & $-L_{3}$ & $-L_{1}$ & $-L_{3}$ & $-L_{3}$ & $-L_{3}$ & $-L_{2}$ & $-L_{1}$ & $L_{1}$ & $-L_{2}$ & $-L_{2}$ & $-L_{1}$ & $L_{1}$ & $L_{2}$ & $-L_{1}$ & 0 & $L_{1}$ & $L_{2}$ & 0 & $L_{1}$ & $L_{2}$ & $L_{1}$ & $L_{2}$ & $L_{3}$ \\
\hline $\mathbf{D}_{7}$ & $-L_{3}$ & $-L_{3}$ & $-L_{3}$ & $-L_{3}$ & $-L_{3}$ & $-L_{3}$ & $-L_{1}$ & $-L_{3}$ & $-L_{3}$ & $-L_{3}$ & $-L_{3}$ & $-L_{1}$ & $L_{1}$ & $-L_{2}$ & $-L_{2}$ & $-L_{1}$ & $-L_{1}$ & $L_{1}$ & $-L_{1}$ & $-L_{1}$ & 0 & $L_{3}$ & 0 & $L_{1}$ & $L_{3}$ & $L_{2}$ & $L_{3}$ & $L_{3}$ \\
\hline $\mathbf{D}_{8}$ & $-L_{3}$ & $-L_{3}$ & $-L_{3}$ & $-L_{3}$ & $-L_{3}$ & $-L_{3}$ & 0 & $-L_{3}$ & $-L_{3}$ & $-L_{3}$ & $-L_{3}$ & $-L_{1}$ & $L_{1}$ & $-L_{2}$ & $-L_{1}$ & $-L_{1}$ & 0 & $L_{2}$ & $-L_{1}$ & 0 & 0 & $L_{2}$ & $L_{1}$ & $L_{1}$ & $L_{2}$ & $L_{3}$ & $L_{3}$ & $L_{3}$ \\
\hline 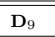 & $-\bar{c}_{3}$ & $\overline{c-L_{3}}$ & $\overline{c-L_{3}}$ & $\bar{c}-L_{3}$ & $\bar{c}-L_{2}$ & $\overline{c-L_{2}}$ & 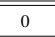 & $-\bar{c}_{3}$ & $-\bar{c}_{3}$ & $\bar{c}-L_{3}$ & $\bar{c}-L_{2}$ & $-L_{1}$ & 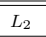 & $-\bar{c}_{3}$ & $\bar{c}-L_{2}$ & $-L_{1}$ & $-L_{1}$ & 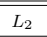 & $-L_{1}$ & 0 & $L_{1}$ & 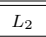 & 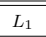 & 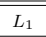 & 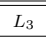 & $\bar{~} L_{1}$ & 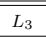 & $L_{3}$ \\
\hline $\mathbf{D}_{10}$ & $-L_{3}$ & $-L_{3}$ & $-L_{3}$ & $-L_{3}$ & $-L_{3}$ & $-L_{2}$ & $-L_{1}$ & $-L_{3}$ & $-L_{3}$ & $-L_{1}$ & $-L_{1}$ & $-L_{1}$ & $L_{1}$ & $-L_{3}$ & $-L_{1}$ & 0 & 0 & $L_{2}$ & $-L_{1}$ & $L_{1}$ & $L_{2}$ & $L_{3}$ & $L_{2}$ & $L_{3}$ & $L_{3}$ & $L_{3}$ & $L_{3}$ & $L_{3}$ \\
\hline $\mathbf{D}_{11}$ & $-L_{3}$ & $-L_{3}$ & $-L_{3}$ & $-L_{3}$ & $-L_{3}$ & $-L_{2}$ & 0 & $-L_{3}$ & $-L_{3}$ & $-L_{3}$ & $-L_{2}$ & 0 & $L_{2}$ & $-L_{3}$ & $-L_{3}$ & 0 & $L_{1}$ & $L_{2}$ & $-L_{1}$ & $L_{1}$ & $L_{2}$ & $L_{3}$ & $L_{1}$ & $L_{2}$ & $L_{3}$ & $L_{2}$ & $L_{3}$ & $L_{3}$ \\
\hline $\mathbf{D}_{12}$ & $-L_{3}$ & $-L_{3}$ & $-L_{3}$ & $-L_{3}$ & $-L_{3}$ & $-L_{3}$ & $-L_{1}$ & $-L_{3}$ & $-L_{3}$ & $-L_{2}$ & $-L_{2}$ & 0 & $L_{1}$ & $-L_{3}$ & $-L_{2}$ & $-L_{2}$ & 0 & $L_{2}$ & $-L_{2}$ & 0 & $L_{2}$ & $L_{2}$ & $L_{2}$ & $L_{2}$ & $L_{3}$ & $L_{3}$ & $L_{3}$ & $L_{3}$ \\
\hline $\mathbf{D}_{13}$ & $-L_{3}$ & $-L_{3}$ & $-L_{3}$ & $-L_{3}$ & $-L_{3}$ & $-L_{2}$ & $-L_{1}$ & $-L_{3}$ & $-L_{3}$ & $-L_{2}$ & $-L_{2}$ & 0 & $L_{1}$ & $-L_{2}$ & $-L_{2}$ & $-L_{1}$ & $L_{1}$ & $L_{2}$ & $-L_{2}$ & $-L_{1}$ & $L_{1}$ & $L_{2}$ & 0 & $L_{2}$ & $L_{3}$ & $L_{3}$ & $L_{3}$ & $L_{3}$ \\
\hline $\mathbf{D}_{14}$ & $-L_{3}$ & $-L_{3}$ & $-L_{3}$ & $-L_{2}$ & $-L_{2}$ & $-L_{2}$ & $-L_{1}$ & $-L_{3}$ & $-L_{3}$ & $-L_{2}$ & $-L_{2}$ & 0 & $L_{2}$ & $-L_{3}$ & $-L_{2}$ & $-L_{2}$ & $L_{1}$ & $L_{2}$ & $-L_{2}$ & $-L_{1}$ & $L_{1}$ & $L_{3}$ & 0 & $L_{2}$ & $L_{3}$ & $L_{3}$ & $L_{3}$ & $L_{3}$ \\
\hline $\mathbf{D}_{15}$ & $-L_{3}$ & $-L_{3}$ & $-L_{3}$ & $-L_{3}$ & $-L_{3}$ & $-L_{3}$ & $-L_{1}$ & $-L_{3}$ & $-L_{3}$ & $-L_{3}$ & $-L_{3}$ & $-L_{2}$ & $L_{1}$ & $-L_{3}$ & $-L_{3}$ & 0 & $L_{1}$ & $L_{1}$ & $-L_{1}$ & $L_{1}$ & $L_{1}$ & $L_{2}$ & $L_{1}$ & $L_{1}$ & $L_{2}$ & $L_{2}$ & $L_{2}$ & $L_{3}$ \\
\hline $\mathbf{D}_{16}$ & $-L_{3}$ & $-L_{3}$ & $-L_{3}$ & $-L_{2}$ & $-L_{2}$ & $-L_{1}$ & 0 & $-L_{3}$ & $-L_{3}$ & $-L_{2}$ & $-L_{2}$ & $-L_{1}$ & $L_{2}$ & $-L_{3}$ & $-L_{2}$ & $-L_{1}$ & $L_{1}$ & $L_{2}$ & $-L_{2}$ & $L_{1}$ & $L_{1}$ & $L_{3}$ & $L_{1}$ & $L_{2}$ & $L_{3}$ & $L_{2}$ & $L_{3}$ & $L_{3}$ \\
\hline
\end{tabular}

described in Section IV. We only indicate the entries of the LUT array (see Table I) that cannot be deduced by symmetry.

\section{REFERENCES}

[1] T. Richardson, "Error floors of LDPC codes," in Proc. 41st Annu. Allerton Conf. Commun., Control, Comput., Monticello, IL, pp. 14261435, Oct. 2003.

[2] B. Vasic, S. K. Chilappagari, D. V. Nguyen, and S. K. Planjery, "Trapping set ontology," Proc. 47th Annu. Allerton Conf. Commun., Control, Comput., Monticello, IL, pp. 1-7, Sep. 2009.

[3] C. A. Kelley and D. Sridhara, "Pseudocodewords of Tanner graphs," IEEE Trans. Inf. Theory, vol. 53, no. 11, pp. 4013-4038, Nov. 2007.

[4] R. Koetter and P. Vontobel, "Graph-covers and iterative decoding of finite-length codes, " Proc. 3rd Int. Conf. on Turbo Codes and Related Topics, Brest, France, pp. 75-82, Sep. 2003.

[5] M. Ivkovic, S. K. Chilappagari, and B. Vasic, "Eliminating trapping sets in low-density parity-check codes by using Tanner graph covers," IEEE Trans. Inf. Theory, vol. 54, no. 8, pp. 3763-3768, Aug. 2008.

[6] F. Mac-Williams and N. Sloane, "The theory of error-correcting codes," North-Holland Mathematical Library, 1978.

[7] S. K. Chilappagari, A. R. Krishnan, and B. Vasic, "LDPC codes which can correct three errors under iterative decoding," Proc. IEEE Inform. Theory Workshop, Porto, Portugal, pp. 406-410, May 2008.

[8] S. K. Planjery, D. Declercq, L. Danjean, and B. Vasic, "Finite alphabet iterative decoders, part I: decoding beyond belief propagation on the binary symmetric channel," accepted to IEEE Trans. Commun., Aug. 2013.

[9] S. K. Planjery, D. Declercq, L. Danjean, and B. Vasic, "Finite alphabet iterative decoders for LDPC codes surpassing floating-point iterative decoders," Electron. Lett., vol. 47, no. 16, pp. 919-921, Aug. 2011.

[10] S. K. Planjery, D. Declercq, S.K. Chilappagari, and B. Vasic, "Multilevel decoders surpassing belief propagation on the binary symmetric channel," in Proc. IEEE Int. Symp. Inform. Theory, Austin, TX, pp. 769-773, Jun. 2010.

[11] L. Danjean, S. K. Planjery, D. Declercq and B. Vasic, "On the selection of finite alphabet iterative decoders for LDPC codes on the BSC,'Proc. Inform. Theory Workshop, Paraty, Brazil, pp. 345-349, Oct. 2011.

[12] M. Tanner, D. Sridhara, and T. Fuja, "A class of group-structured LDPC codes," Proc. 5th Int. Symp. Commun. Theory App., Ambleside, England, Jul. 2001.

[13] D. Declercq, L. Danjean, S. K. Planjery and B. Vasic, "Finite alphabet iterative decoding (FAID) of the $(155,64,20)$ Tanner code," in Proc. Int. Symp. Turbo Codes Iter. Inform. Process., Brest, France, pp. 11-15, Sep. 2010.

[14] M. Fossorier, "Quasi-cyclic low-density parity-check codes from circulant permutation matrices," IEEE Trans. Inf. Theory, vol. 50, no. 8, pp. 1788-1793, Aug. 2004.
[15] S. K. Chilappagari, S. Sankaranarayanan, and B. Vasic, "Error floors of LDPC codes on the binary symmetric channel," Proc. IEEE Int. Conf. Commun., Istanbul, Turkey, pp. 1089-1094, Jun. 2006.

[16] Richard M. Karp (1972), Reducibility among combinatorial problems, R. E. Miller and J. W. Thatcher (editors). Complexity of Computer Computations. New York: Plenum. pp. 85-103.

[17] M. Tanner, D. Sridhara, A. Sridharan, T. Fuja and D. Costello Jr., "LDPC block and convolutional codes based on circulant matrices," IEEE Trans. Inf. Theory, vol. 50, no 12, pp. 2966-2984, Dec. 2004.

[18] L. Dolecek, Z. Zhang, V. Anatharam, M. Wainwright and B. Nikolic, "Analysis of absorbing sets and fully absorbing sets of array-based LDPC codes," IEEE Trans. Inf. Theory, vol. 56, no. 1, pp. 181-201, Jan. 2010.

[19] S. K. Chilappagari, M. Chertkov, and B. Vasic, "An efficient instanton search algorithm for LP decoding of LDPC codes over the BSC," IEEE Trans. Inf. Theory, vol. 57, no. 7, pp. 4417-4426, Jul. 2011.

[20] D. Declercq and M. Fossorier, "Improved impulse method to evaluate the low-weight profile of sparse binary linear codes," Proc. IEEE Int. Symp. Inform. Theory, Toronto, Canada, pp. 1963-1967, Jul. 2008.

[21] S. Abu-Surra, D. Declerq, D. Divsalar, and W. Ryan, "Trapping set enumerators for specific LDPC codes," in Proc. Inform. Theory App. Workshop, San Diego, CA, pp.1-5, Feb. 2010.

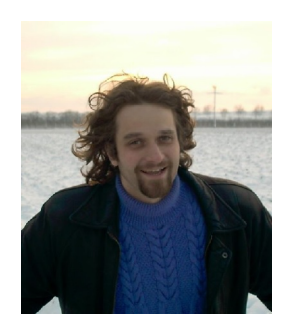

David Declercq was born in June 1971. He graduated his PhD in Statistical Signal Processing 1998 from the University of Cergy-Pontoise, France. He is currently full professor at the ENSEA in CergyPontoise. He is the general secretary of the National GRETSI association, and Senior member of the IEEE. He is currently the recipient of junior position at the "Institut Universitaire de France". His research topics lie in digital communications and errorcorrection coding theory. He worked several years on the particular family of LDPC codes, both from the code and decoder design aspects. Since 2003, he developed a strong expertise on non-binary LDPC codes and decoders in high order Galois fields GF(q). A large part of his research projects are related to non-binary LDPC codes. He mainly investigated two aspects: (i) the design of GF(q) LDPC codes for short and moderate lengths, and (ii) the simplification of the iterative decoders for GF(q) LDPC codes with complexity/performance tradeoff constraints. David Declercq published more than 30 papers in major journals (IEEE-Trans. Commun., IEEE-Trans. Inf. Theo., Commun. Letters, EURASIP JWCN), and more than 100 papers in major conferences in Information Theory and Signal Processing. 


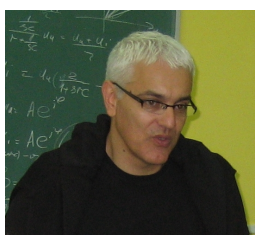

Bane Vasic is a Professor of Electrical and Computer Engineering and Mathematics at the University of Arizona. He is affiliated with BIO5, the Institute for Collaborative Beoresearch, and is a Director of the Error Correction Laboratory. Current sponsors and collaborators of his laboratory include NSF, DARPA, IDEMA, INSIC NASA, LANL, Seagate Technology, IBM, Hitachi, Toshiba, LSI Corp., and Bell Laboratories.

Dr. Vasic is an inventor of the soft error-event decoding algorithm, and the key architect of a detector/decoder for Bell Labs magnetic recording read channel chips which were regarded as the best in industry. Different variants of this algorithm were implemented in virtually all magnetic hard drives. His pioneering work on structured low-density parity check (LDPC) error correcting codes and invention of codes has enabled low-complexity iterative decoder implementations. Structured LDPC codes are today adopted in a number of communications standards and in extremely high-density magnetic storage systems. He was also involved in a DVD Copy Protection Standardization Group sponsored by the DVD Industry Consortium and Hollywood Movie Studios.

Dr. Vasic is known for his theoretical work in error correction coding theory and codes on graphs which has led to analytical characterization of the hard decision iterative decoders of LDPC codes, and design of codes with best error-floor performance known today.

$\mathrm{He}$ is an IEEE Fellow and da Vinci Fellow.

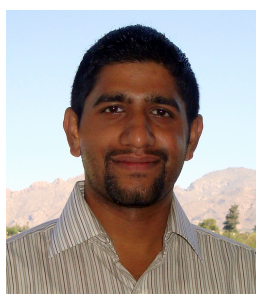

Shiva Kumar Planjery received a B.Tech in Electrical Engineering from the Indian Institute of Technology - Madras, Chennai, India, in 2005, and a M.A.Sc. in Electrical Engineering from the University of Victoria, BC, Canada, in 2007. Under a dual degree program, he received a Ph.D. from the University of Cergy-Pontoise, Cergy-Pontoise, France, in Dec. 2012, with a specialization in communication and information theory, and a Ph.D. from the University of Arizona, Tucson, AZ, in Electrical Engineering in Aug. 2013. His current research interests include error control coding, information theory, and digital communications with specific focus on the design and analysis of lowcomplexity iterative decoding algorithms for LDPC codes.

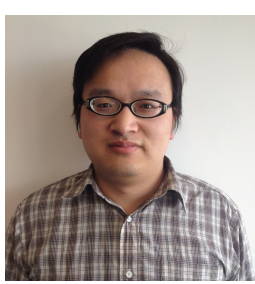

Erbao Li was born in Anhui, China. He received the B.S.E.E. degree from Northwestern Polytechnical University, Xi' an, China, in 2005, and the M.S.E.E. degree from National University of Defense Technology, Changsha, China, in 2007, and the Ph.D. degree in communication and information theory from the University of Cergy-Pontoise, Paris, in 2012. He is currently a post-doc in ETIS ENSEA/univ. CergyPontoise/CNRS. His research interests include errorcontrol coding for digital communications and iterative decoding algorithms, especially in complexity reduced algorithms for decoding Non-binary LDPC codes. 